\title{
Annette Vowinckel, Agenten der Bilder. Fotografisches Handeln im 20. Jahrhundert
}

\author{
Bérénice Zunino
}

\section{OpenEdition}

Journals

Édition électronique

URL : http://journals.openedition.org/ifha/9302

DOI : $10.4000 /$ ifha.9302

ISSN : 2198-8943

Éditeur

IFRA - Institut franco-allemand (sciences historiques et sociales)

Référence électronique

Bérénice Zunino, «Annette Vowinckel, Agenten der Bilder. Fotografisches Handeln im 20. Jahrhundert», Revue de l'IFHA [En ligne], Date de recension, mis en ligne le 20 juin 2018, consulté le 24 septembre 2020. URL : http://journals.openedition.org/ifha/9302 ; DOI : https://doi.org/10.4000/ifha.9302

Ce document a été généré automatiquement le 24 septembre 2020

(CIFHA 


\section{Annette Vowinckel, Agenten der Bilder. Fotografisches Handeln im 20. Jahrhundert}

Bérénice Zunino

\section{RÉFÉRENCE}

Annette Vowinckel, Agenten der Bilder. Fotografisches Handeln im 20. Jahrhundert, Göttingen: Wallstein, 2016, 480 p., 34,90€ 
Dans son étude de belle facture comportant de nombreuses illustrations en couleur, Annette Vowinckel, qui codirige le département d'«Histoire contemporaine de la société des médias et de l'information » au Zentrum für Zeithistorische Forschung en coopération avec Jürgen Danyel depuis 2014, a réussi à relever un défi de taille: écrire une histoire de la photographie au $\mathrm{XX}^{\mathrm{e}}$ siècle en se focalisant sur son inscription dans le champ politique et ses fonctions dans la formation d'une «opinion publique visuelle globale» (p.31). De ce fait, même s'il touche de près à l'histoire des photographies de presse, cet ouvrage dépasse le cadre de l'histoire de la presse et du journalisme, comme celui des études visuelles. S'intéressant à la fois à la

Annette Vowinckel Agenten der Bilder Fotografisches Handeln im 20. Jahrhundert

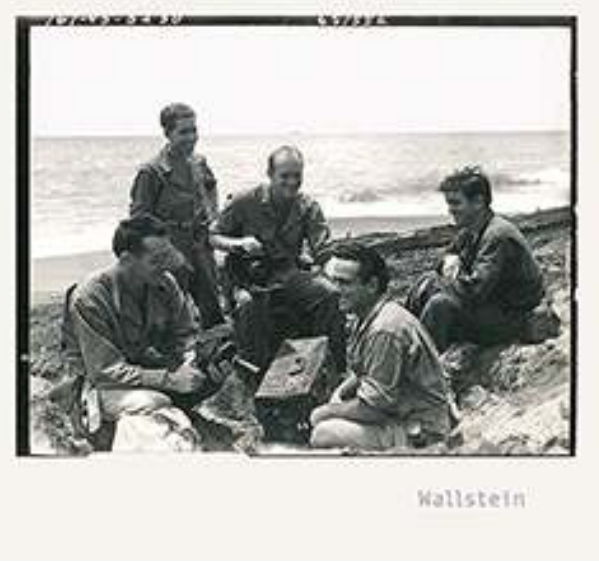
production et aux usages des photographies, A. Vowinckel conçoit son étude comme une «contribution à l'histoire de la photographie » et comme " un ouvrage consacré aux changements du politique au $\mathrm{XX}^{\mathrm{e}}$ siècle » (p. 8). Ainsi, cette étude pourra être fort utile tant aux spécialistes de la presse et aux historiens du visuel qu'aux historiens de la RDA (dont il est question dans plusieurs chapitres) et aux spécialistes des conflits. On remarque, de fait, que les guerres ont joué un rôle déterminant dans la constitution de ce vaste réseau d'images structurant l'opinion publique.

À la croisée de l'histoire et de la communication politique, directement inspirée des travaux de Herfried Münkler et de Marion G. Müller, l'auteure tend à infléchir la thèse de l'« acte imagé » («Bildakt », Horst Bredekamp) qui a inspiré tant d'études en histoire visuelle: considérées comme des "êtres vivants engendrant des faits", les images seraient «formatrices d'histoire» (prägend) et moteur de l'action dans le champ politique et social. Or, en attribuant un pouvoir performatif aux images, H. Bredekamp substituerait les images aux êtres humains pourtant acteurs de l'histoire (Bernd Stiegler).

Dans une approche transnationale, A. Vowinckel ne s'est donc pas (seulement) penchée sur les images elles-mêmes, mais a cherché à rétablir le rôle de leurs " agents » (p. 8), autrement dit les acteurs impliqués dans la chaîne de production et de publication des photographies qui forgent par-là la «puissance» (agency) des images. Définissant l'«agir photographique» ("fotografisches Handeln») comme une "forme spéciale de l'agir imagé qui développe sa pleine puissance et trouve toute sa place dans la société grâce aux techniques de reproduction de masse" (p.14) et s'appuyant sur une définition politique de l'opinion publique appréhendée dans ses variations historiques, géographiques et politiques dans la tradition d'Habermas, l'auteure cherche à montrer que «la formation de l'opinion publique est également induite par la circulation d'images » (p. 14). La " photographie documentaire », en particulier, au centre de cette étude, peut être aisément utilisée à cette fin comme « argument visuel » (p. 16). 
Sur cette base théorique, A. Vowinckel livre une analyse des différents acteurs de la chaine de production photographique: photojournalistes, éditeurs visuels (Bildredakteure), agences photographiques, agences de presse, etc. Elle aborde également les aspects juridiques des droits et de l'utilisation des images, ainsi que les questions éthiques que posent les photographies (de guerre, de violences et de personnalités publiques). L'ouvrage se divise en cinq chapitres : "L'émergence d'une opinion publique visuelle globale ", «Les agents des images : catégories de métiers », "Photographes et photographies au service de l'État ", "Contrôle et utilisation des images » et enfin un chapitre consacré à différentes études de cas aussi variées que le photojournalisme en Afrique, la «subversion photographique» en RDA (p. 327), les traditions visuelles fascistes et antifascistes et l'usage de la photographie par les personnalités politiques. Les bornes chronologiques retenues, sur lesquelles A. Vowinckel revient au cours de son étude, correspondent à la période allant des années 1930 aux années 1980.

L'auteure distingue préalablement cinq cas idéaltypiques dans lesquels les photographies peuvent être utilisées à ces fins argumentatives: 1) formation d'une opinion publique visuelle, depuis que les hommes politiques, dans les années 1930, ne sont plus seulement attentifs à leurs actes, mais aussi à leur image ; 2) agitation, propagande et Public Relations ; 3) appel à la solidarité dans des contextes de guerre et de violences - fonction qui s'est largement développée depuis la guerre d'Espagne; 4) «subversion photographique » (p.19), utilisée notamment dans des régimes nondémocratiques, comme en RDA ; 5) image comme preuve, mise à profit dans des contextes militaires (prises de vue aériennes) ou scientifiques (le cas controversé de la Wehrmachtausstellung d'Hambourg en est un exemple). On peut s'étonner que la structure de l'ouvrage ne repose pas sur ces catégories, mais celles-ci sont remobilisées comme outils d'analyse dans différents passages, en premier lieu dans le dernier chapitre consacré aux études de cas.

L'analyse de l'émergence d'une opinion publique visuelle globale dans le premier chapitre de l'ouvrage est d'autant plus intéressante qu'elle nuance grandement, comme d'autres études récentes (T. Gervais), l'idée (forgée par les photoreporters eux-mêmes dans leurs autobiographies) selon laquelle le photojournalisme serait une apparition européenne, en premier lieu allemande. D'après l'auteure, cette opinion publique visuelle globale va de pair avec la "globalisation du commerce des photographies " (p. 39) et la formation d'un « réseau d'images global» (p. 49), et a été rendue possible grâce à «l'expansion d'agences de presse, qui se développèrent à la manière d'un réseau de métro ou d'une toile d'araignée » (p. 54). À partir de l'exemple de l'agence américaine pionnière Associated Press (AP, 1846, New York), A. Vowinckel retrace l'expansion du modèle d'organisation américain en Europe, alors qu'au début du $\mathrm{XX}^{\mathrm{e}}$ siècle, de ce côté de l'Atlantique, les agences photographiques (Fotoagenturen) qui dominaient le marché n'étaient pas rattachées à des agences de presse (Nachrichtenagenturen). Progressivement, jusqu'aux années 1960, AP et ses «services d'images» (Bilderdienste) - ainsi que d'autres agences de presse - s'étendent dans une quinzaine de pays européens avant de s'implanter en Afrique, par le biais du commerce colonial et de l'amélioration des techniques de transmission. La guerre du Vietnam favorise parallèlement leur expansion en Asie orientale.

Dans le but d'écrire «une histoire de l'opinion publique visuelle centrée sur les acteurs » (p. 57), A. Vowinckel développe dans les chapitres suivants les trois résultats 
principaux de ses recherches. Le premier aspect, certainement le plus novateur, s'axe autour des fonctions des éditeurs visuels et de leur place dans la chaîne de production des photographies de presse ainsi que de leurs rapports - de coopération, mais aussi de concurrence - avec les photojournalistes, dont la typologie est mieux connue. À partir des manuels conçus pour les éditeurs visuels, de documents d'archives et d'autobiographies de reporters (de guerre, essentiellement), elle dresse, d'après le modèle wébérien, un idéal-type de ces deux catégories professionnelles.

Se dégagent des aspects communs dans ces deux typologies : photojournalistes comme éditeurs visuels sont majoritairement des hommes, célibataires sans enfants, autodidactes immigrés et/ou d'origine juive qui se tournent vers le photojournalisme par nécessité pécuniaire. L'un des exemples paradigmatiques est Erich Salomon, juriste de formation, qui s'est formé seul au métier de photographe et a engagé une coopération avec la Berliner Illustrierte Zeitung après avoir été fortement touché par la crise économique de 1929. Contrairement aux photojournalistes, qui soignent leur image d'aventuriers au service d'une cause pacifiste et humanitaire, les éditeurs visuels jouissent de conditions de travail moins précaires et restent souvent anonymes. On peut malgré tout citer quelques grands noms pionniers: Stefan Lorant (Münschner Illustrierte Zeitung), Tom Hopkinson (Picture Post), Wilson Hicks (Life) et Maria Eisner, fondatrice de l'agence de presse parisienne Alliance Photo. A. Vowinckel met en lumière les aspects visuels méconnus du processus de rédaction photojournalistique composition, agencement des maquettes, etc. -, qui, comme elle le souligne, relève de sélections et de choix effectués par ces acteurs de la presse qui accordent aux images une agency.

Le deuxième résultat s'axe autour du rôle des États et des gouvernements dans la formation des opinions visuelles. Ce volet s'inscrit dans la lignée des études sur la propagande - dont A. Vowinckel présente les différentes acceptions et les difficultés méthodologiques qu'elles soulèvent - et le contrôle de l'image. Les exemples d'institutions militaires (unités photographiques de la US Army Signal Corps, Propagandakompagnien de la Wehrmacht, etc.) et civiles (projet photographique de la Farm Security Administration, célèbre pour son cliché de la "Migrant Mother ", double exposition consacrée au Mur de Berlin de 1961-1962, méconnue, organisée à l'initiative de l'USIA, du ministère des Affaires étrangères ouest-allemand et de l'office fédéral de la presse) montrent que l'ampleur des photographies publiées dans la presse quotidienne et magazine à l'initiative des autorités publiques est sous-estimée. Dans des contextes de crise ou de conflit, États et gouvernements misent sur l'information, le contrôle de l'opinion ou, dans le cas de la double exposition sur le Mur de Berlin par exemple, sur la " polarisation du public » (p. 232) durant la Guerre froide.

Outre les mesures émanant de la sphère militaire ou politique, les exemples les plus intéressants abordés par l'auteure sont les cas hybrides : peu désireux de dévoiler leur implication dans une entreprise de production photographique, les États chapeautent des institutions tout en restant en retrait. Parmi elles, on peut citer l'agence de presse Transocean à l'époque du Kaiserreich allemand ou l'agence photographique Zentralbild rattachée à l'agence de presse officielle de RDA. De ces analyses, A. Vowinckel tire deux conclusions principales. Premièrement, quel que fût leur système politique, les États ont su utiliser la production photographique dans le but de former une opinion publique visuelle. Deuxièmement, l'auteure constate que les photographies furent moins soumises au contrôle et à la censure que d'autres sources (lettres, romans, mais 
aussi films). Ce résultat, qui confirme d'autres thèses récentes (voir par exemple l'étude de Christine Brocks sur les cartes postales de 1914-1918: Die Bunte Welt des Krieges), aurait toutefois mérité d'être étayé par une analyse iconologique lorsqu'A. Vowinckel affirme que ce manque de préoccupation des autorités publiques pour ces images serait dû à « l'ambivalence de la photographie » (p. 288).

Les réflexions de l'auteure quant aux débuts du photojournalisme et à son âge d'or présumé constituent le troisième résultat de ses investigations. Conformément aux nouvelles tendances de la recherche qui ne se cantonnent plus au lieu commun d'un âge d'or du photojournalisme dans les années 1920, elle aborde les débuts du photojournalisme professionnel à la fin du XIXe siècle et conteste l'idée d'un " déclin du photojournalisme dû à l'essor de la télévision» (p. 430) dans les années 1970 qui s'expliquerait par la place démesurée accordée à Life et à sa disparition en 1972. Bien qu'elle souligne que «le récit de l'essor de la photographie de reportage dans les années $1920 »$ (p.430) a été forgé par les photojournalistes eux-mêmes, elle reste convaincue que cette décennie représente la césure principale dans l'histoire du photojournalisme. À cet égard, on peut regretter que les travaux de Vincent Lavoie et le dernier ouvrage de Thierry Gervais, La Fabrique de l'information visuelle (2016) ne figurent pas dans la bibliographie, mais ce dernier a dû paraître peu de temps avant la présente étude.

On pourrait également discuter l'affirmation selon laquelle l'instrumentalisation politique de la photographie serait plus prononcée que celle d'autres genres et supports iconographiques; des gravures au temps de la Réforme aux affiches et cartes postales de la Première Guerre mondiale en passant par les dessins politiques des Révolutions de 1830 et 1848 , les "arguments visuels" de toute nature n'ont pas manqué d'être mobilisés depuis que les techniques de reproduction le permettent. Par ailleurs, dans la sous-partie consacrée à l'usage de la photographie à des fins d'enquête policière (p. 311), on peut regretter qu'il ne soit pas fait mention du rôle de la Commune de Paris dans l'essor de la photographie criminelle en France. Enfin, la définition d'icônes, « qui veut tout simplement dire qu'elles sont très vraisemblablement reconnues par la plupart des gens et qu'elles peuvent être associées à un contenu historique ou thématique » (p.52) en raison de leurs nombreuses occurrences médiatiques, pourrait être également sujette à débat. De fait, des historiens du visuel n'y voient pas une simple multiplication d'occurrences facilitée par des choix éditoriaux (p. 53), mais un processus d'allégorisation par lequel une image passe du statut de document d'actualité à un médium narratif historique et acquiert une dimension symbolique (André Gunthert, Camille Rouquet). Toutefois, au vu de l'ampleur et de la qualité des investigations menées par A. Vowinckel et des résultats novateurs et stimulants auxquels elle aboutit - surtout concernant les éditeurs visuels -, on ne peut que recommander la lecture de cet ouvrage remarquable, et parier sur le fait qu'il inspirera d'autres études sur les photographies de presse, leur production et leurs usages. 
INDEX

Index chronologique : Époque contemporaine

Thèmes : Histoire de la culture, Histoire militaire, Histoire sociale

\section{AUTEURS}

BÉRÉNICE ZUNINO

Université de Franche-Comté, EA 3224 - Centre de Recherches Interdisciplinaires et Transculturelles, membre partenaire du SIRICE 